\title{
Leading-particle suppression in high energy nucleus-nucleus collisions
}

\author{
A. Dainese ${ }^{a, *}$, C. Loizides ${ }^{b, \dagger}$ and G. Paic $c^{c, \ddagger}$ \\ ${ }^{a}$ Università degli Studi di Padova and INFN, via Marzolo 8, 35131 Padova, Italy \\ ${ }^{b}$ Institut für Kernphysik, August-Euler-Str. 6, D-60486 Frankfurt am Main, Germany \\ ${ }^{c}$ Instituto de Ciencias Nucleares, UNAM, Mexico City, Mexico
}

18th June 2004

\begin{abstract}
Parton energy loss effects in heavy-ion collisions are studied with the Monte Carlo program PQM (Parton Quenching Model) constructed using the BDMPS quenching weights and a realistic collision geometry. The merit of the approach is that it contains only one free parameter that is tuned to the high- $p_{\mathrm{t}}$ nuclear modification factor measured in central $\mathrm{Au}-\mathrm{Au}$ collisions at $\sqrt{s_{\mathrm{NN}}}=200 \mathrm{GeV}$. Once tuned, the model is coherently applied to all the high- $p_{\mathrm{t}}$ observables at $200 \mathrm{GeV}$ : the centrality evolution of the nuclear modification factor, the suppression of the away-side jet-like correlations, and the azimuthal anisotropies for these observables. Predictions for the leading-particle suppression at nucleon-nucleon centre-of-mass energies of 62.4 and $5500 \mathrm{GeV}$ are calculated. The limits of the eikonal approximation in the BDMPS approach, when applied to finite-energy partons, are discussed.
\end{abstract}

\footnotetext{
*andrea.dainese@pd.infn.it

†loizides@ikf.uni-frankfurt.de

†guypaic@nuclecu.unam.mx
} 


\section{Introduction}

High-momentum leading-particle suppression in nucleus-nucleus (AA) with respect to proton-proton collisions is regarded as one of the major discoveries at the Relativistic Heavy Ion Collider (RHIC), Brookhaven. In $\mathrm{Au}-\mathrm{Au}$ collisions at centre-of-mass energy $\sqrt{s_{\mathrm{NN}}}=200 \mathrm{GeV}$ per nucleon-nucleon (NN) pair, the two experiments with high transverse momentum, $p_{\mathrm{t}}$, capabilities, PHENIX and STAR, have measured:

- the suppression of single particles at high $p_{\mathrm{t}}(\gtrsim 4 \mathrm{GeV})$, quantified via the nuclear modification factor

$$
R_{\mathrm{AA}}\left(p_{\mathrm{t}}\right) \equiv \frac{1}{\text { binary NN coll. }} \times \frac{\mathrm{d} N_{\mathrm{AA}} / \mathrm{d} p_{\mathrm{t}}}{\mathrm{d} N_{\mathrm{pp}} / \mathrm{d} p_{\mathrm{t}}},
$$

which would be equal to unity if the AA collision was a mere superposition of independent NN collisions; instead, at high $p_{\mathrm{t}} R_{\mathrm{AA}}$ is found to decrease from peripheral to central events, down to $\approx 0.2$ in head-on collisions 11, 2]; the suppression is the same for charged hadrons and neutral pions for $p_{\mathrm{t}} \gtrsim 5 \mathrm{GeV}$;

- the disappearance, in central collisions, of jet-like correlations in the azimuthally opposite side of a high- $p_{\mathrm{t}}$ leading particle [3]

- the absence of such effects in d-Au collisions at the same energy [4, 5].

These observations can be naturally explained in terms of attenuation (quenching) of energetic partons produced in initial hard scattering processes, as a consequence of the interaction with the dense QCD medium expected to be formed in high energy heavy-ion collisions. Several theoretical works exist on the subject 6, 7, 8, 9, 10, 11. Most of them implement the idea of parton energy loss due to medium-induced gluon radiation.

In our Monte Carlo program PQM (Parton Quenching Model) we combine a state-of-the-art calculation of parton energy loss [1] and a realistic description of the collision geometry, which was recently proven to play an important role [12. Our approach allows to study and compare to RHIC data the transverse momentum and centrality dependence of single-hadron and di-hadron correlation suppressions, as well as the 'energy-loss induced' azimuthal anisotropy of particle production in non-central collisions. The model has one single parameter that sets the scale of the energy loss. Once the parameter is fixed on the basis of the data at $\sqrt{s_{\mathrm{NN}}}=200 \mathrm{GeV}$, we scale it to different energies assuming its proportionality to the expected volume-density of gluons, as argued in Ref. [13. We then apply the same approach to calculate the nuclear modification factors at intermediate RHIC energy, $\sqrt{s_{\mathrm{NN}}}=62.4 \mathrm{GeV}$, and at LHC energy, $\sqrt{s_{\mathrm{NN}}}=5.5 \mathrm{TeV}$. Since we 
do not include so-called initial-state effects, such as nuclear modification of the parton distribution functions and parton intrinsic transverse-momentum broadening, we restrict our study to the high- $p_{\mathrm{t}}$ region, above $4-5 \mathrm{GeV}$ at RHIC energies and above $10 \mathrm{GeV}$ at LHC energy, where these effects are expected to be small (less than $10 \%$ on $R_{\mathrm{AA}}$ ) [14, 15].

\section{Parton energy loss and collision geometry}

For the calculation of in-medium parton energy loss, we use the quenching weights in the multiple soft scattering approximation, which were derived in Ref. [1] in the framework of the 'BDMPS' (Baier-Dokshitzer-MuellerPeigné-Schiff) formalism [7].

In a simplified picture, an energetic parton produced in a hard collision undergoes, along its path in the dense medium, multiple scatterings in a Brownian-like motion with mean free path $\lambda$, which decreases as the medium density increases. In this multiple scattering process, the gluons in the projectile-parton wave function pick up transverse momentum $k_{\mathrm{t}}$ with respect to its direction and they may eventually decohere and be radiated.

The scale of the energy loss is set by the maximum energy of the radiated gluons

$$
\omega_{c}=\hat{q} L^{2} / 2,
$$

which depends on the in-medium path length $L$ of the projectile parton and on the BDMPS transport coefficient of the medium, $\hat{q}$. The transport coefficient is defined as the average medium-induced transverse momentum squared transferred to the projectile per unit path length, $\hat{q}=$ $\left\langle q_{\mathrm{t}}^{2}\right\rangle_{\text {medium }} / \lambda$ [11]. For a static medium it is time-independent.

Differently from the original BDMPS calculation [7], in Ref. [1] the transverse momentum $k_{\mathrm{t}}$ of a radiated gluon is kinematically bound to be smaller than its energy $\omega$; this imposes a constraint on the emission probability via the dimensionless quantity

$$
R=\omega_{c} L=\hat{q} L^{3} / 2 .
$$

The BDMPS case corresponds to $R \rightarrow \infty$ and it can be recovered by considering an infinitely-extended medium $\left(L \rightarrow \infty\right.$ for fixed finite $\left.\omega_{c}\right)$ [1].

The two parameters $\omega_{c}$ and $R$ determine the energy distribution of radiated gluons, $\omega \mathrm{d} I / \mathrm{d} \omega$. While $\omega_{c}$ sets the scale of the distribution, $R$ controls its shape in the region $\omega \ll \omega_{c}$. In the limit $R \rightarrow \infty$ the distribution, for $\omega<\omega_{c}$, is of the form:

$$
\lim _{R \rightarrow \infty} \omega \frac{\mathrm{d} I}{\mathrm{~d} \omega} \simeq \frac{2 \alpha_{s} C_{R}}{\pi} \sqrt{\frac{\omega_{c}}{2 \omega}}
$$


where $C_{R}$ is the QCD coupling factor (Casimir factor), equal to $4 / 3$ for a quark projectile and to 3 for a gluon projectile.

The integral of the energy distribution estimates the average energy loss of the parton:

$$
\langle\Delta E\rangle=\int_{0}^{\omega_{c}} \omega \frac{\mathrm{d} I}{\mathrm{~d} \omega} \mathrm{d} \omega \propto \alpha_{s} C_{R} \omega_{c} \propto \alpha_{s} C_{R} \hat{q} L^{2} .
$$

The average energy loss is: proportional to $\alpha_{s} C_{R}$ and, thus, larger by a factor $9 / 4=2.25$ for gluons than for quarks; proportional to the transport coefficient of the medium; proportional to $L^{2}$; independent of the parton initial energy $E$. It is a general feature of all parton energy loss calculations [7, 9, 11] that the energy distribution (41) does not depend on E. Depending on how the kinematic bounds are taken into account, the resulting $\Delta E$ is $E$-independent [7] or depends logarithmically on $E[9]$. However, there is always a stronger intrinsic dependence of the radiated energy on the initial energy, determined by the fact that the former cannot be larger than the latter, $\Delta E \leq E$. This point will be further discussed in Section 3 .

The probability $P(\Delta E)$ that a hard parton radiates the energy $\Delta E$ due to scattering in spatially-extended QCD matter is known as the quenching weight [16. In Ref. 11] the weights are calculated on the basis of the BDMPS formalism for quarks and gluons as a function of the two parameters $\omega_{c}$ and $R$ and they are given as:

$$
P(\Delta E)=p_{0} \delta(\Delta E)+p(\Delta E)
$$

In this expression, the discrete weight $p_{0}$ is the probability to have no mediuminduced gluon radiation and the continuous weight $p(\Delta E)$ is the probability to radiate an energy $\Delta E$, if at least one gluon is radiated.

It has been shown [1] that a simple scaling law exists, which translates the gluon energy distribution for an expanding medium with a timedecreasing $\hat{q}(t)$ into an equivalent distribution for a static medium, with $\langle\hat{q}\rangle=$ constant, using

$$
\langle\hat{q}\rangle=\frac{2}{L^{2}} \int_{\xi_{0}}^{L+\xi_{0}}\left(\xi-\xi_{0}\right) \hat{q}(\xi) \mathrm{d} \xi,
$$

where $\xi_{0} \sim 10^{-1} \mathrm{fm} \ll L$ is the formation time of the expanding system.

Due to the fact that $\hat{q}$ and $L$ are two more intuitively and physically meaningful parameters, in all the previous applications [11, 19, 13] the natural $\left(R, \omega_{c}\right)$-dependence of the quenching weights was 'translated' into a $(\hat{q}, L)$ dependence, via Eqs. (2) and (3). The common approach was to fix a value 
for the transport coefficient, the same for all produced partons, and then either use a constant length [1] or calculate a different length for each parton according to a description of the collision geometry [19, 13]. However, this approach is not optimal because (a) there is no unique and exact definition of the in-medium path length when a realistic nuclear density profile is used, as pointed out in Ref. [19], and (b) it is not possible to take into account that the medium density is not constant over the whole nucleus-nucleus overlap region but rather decreasing from the centre to the periphery.

In order to overcome these limits, we adopt a new approach in which the two parameters $\omega_{c}$ and $R$ that determine the quenching weights are computed on a parton-by-parton basis, taking into account both the amount (length) and the density (transport coefficient) of the matter traversed by the parton.

Starting from Eqs. (2) and (17), we define the effective quantity

$$
\left.\omega_{c}\right|_{\text {effective }}=\frac{1}{2}\langle\hat{q}\rangle L^{2}=\int_{0}^{\infty} \xi \hat{q}(\xi) \mathrm{d} \xi,
$$

which on the r.h.s. does not explicitly depend on $L$. For a step-function distribution $\hat{q}(\xi)=\hat{q}_{0} \theta(L-\xi)$, Eq. (8) coincides with Eq. (2). Similarly, we define

$$
\left.\langle\hat{q}\rangle L\right|_{\text {effective }}=\int_{0}^{\infty} \hat{q}(\xi) \mathrm{d} \xi
$$

and

$$
\left.R\right|_{\text {effective }}=\frac{2\left(\left.\omega_{c}\right|_{\text {effective }}\right)^{2}}{\left.\langle\hat{q}\rangle L\right|_{\text {effective }}} .
$$

Using the definitions in Eqs. (8)-(10) there is a natural way to incorporate the collision geometry in the calculation of parton energy loss via the 'local' transport coefficient $\hat{q}(\xi)$.

\section{Leading-particle suppression procedure}

In PQM we calculate the leading-particle suppression in nucleus-nucleus collisions in a Monte Carlo approach. The transverse momentum distributions of charged hadrons are generated by means of the following chain:

1. generation of a parton, quark or gluon, with $p_{\mathrm{t}}>3 \mathrm{GeV}$, using the PYTHIA event generator 20] in pp mode with CTEQ 4L parton distribution functions [21]; the $p_{\mathrm{t}}$-dependence of the quarks-to-gluons ratio is taken from PYTHIA;

2. determination of the two input parameters, $\omega_{c}$ and $R$, for the calculation of the quenching weights, i.e. the energy-loss probability distribution $P(\Delta E)$; 
3. sampling of an energy loss $\Delta E$ according to the quenching weights and definition of the new parton transverse momentum, $p_{\mathrm{t}}^{\prime}=p_{\mathrm{t}}-\Delta E$;

4. (independent) fragmentation of the parton to a hadron using the leadingorder Kniehl-Kramer-Pötter (KKP) fragmentation functions 22].

Steps 2 and 3 are explained in detail in the following paragraphs. Quenched and unquenched $p_{\mathrm{t}}$ distributions are obtained including or excluding the third step of the chain. The nuclear modification factor $R_{\mathrm{AA}}\left(p_{\mathrm{t}}\right)$ is given by their ratio. The hadrons $p_{\mathrm{t}}$ distribution we obtain without energy loss at $\sqrt{s}=$ $200 \mathrm{GeV}$ agrees in shape with that measured for neutral pions in pp collisions by PHENIX [23].

\section{Determination of $\omega_{c}$ and $R$}

We define the collision geometry in the $(x, y)$ plane transverse to the beam direction $z$, in which the centres of two nuclei A and B colliding with impact parameter $b$ have coordinates $(-b / 2,0)$ and $(b / 2,0)$. Using the Glauber model [17] to describe the geometry of the collision, we assume (a) the distribution of parton production points in the transverse plane and (b) the transverse density of the medium both to be proportional to the $b$-dependent product $T_{\mathrm{A}} T_{\mathrm{B}}(x, y ; b) \equiv T_{\mathrm{A}}(x, y) \times T_{\mathrm{B}}(x, y)$ of the thickness functions of the two nuclei. The nuclear thickness function is defined as the $z$-integrated Wood-Saxon nuclear density profile: $T_{\mathrm{i}}(x, y)=\int \mathrm{d} z \rho_{i}^{\mathrm{WS}}(x, y, z)$. The parameters of the Wood-Saxon profile for different nuclei are tabulated from data [18. Note that $T_{\mathrm{A}} T_{\mathrm{B}}(x, y ; b)$ estimates the transverse density of binary NN collisions, $\rho_{\text {coll }}(x, y ; b)$, modulo the inelastic NN cross section.

We consider only partons produced at central rapidities and we can then assume they propagate in the transverse plane $\left(E \approx p \approx p_{\mathrm{t}}\right)$. For a parton with production point $\left(x_{0}, y_{0}\right)$ and azimuthal propagation direction $\left(u_{x}, u_{y}\right)$ (which is sampled uniformly), we define the 'local' transport coefficient along the path of the parton inside the overlap region of the nuclei as:

$$
\hat{q}(\xi ; b)=k \times T_{\mathrm{A}} T_{\mathrm{B}}\left(x_{0}+\xi u_{x}, y_{0}+\xi u_{y} ; b\right)
$$

where $k$ is a constant that sets the scale of the transport coefficient. We compute the two integrals $I_{0}$ and $I_{1}$ (Eqs. (9) and (8) )

$$
I_{n} \equiv \int_{0}^{\infty} \xi^{n} \hat{q}(\xi ; b) \mathrm{d} \xi \quad n=0,1,
$$

which determine the energy-loss probability distribution $P(\Delta E)$ through (see Section 2):

$$
\omega_{c}=I_{1} \quad \text { and } \quad \mathrm{R}=2 \mathrm{I}_{1}^{2} / \mathrm{I}_{0} \text {. }
$$



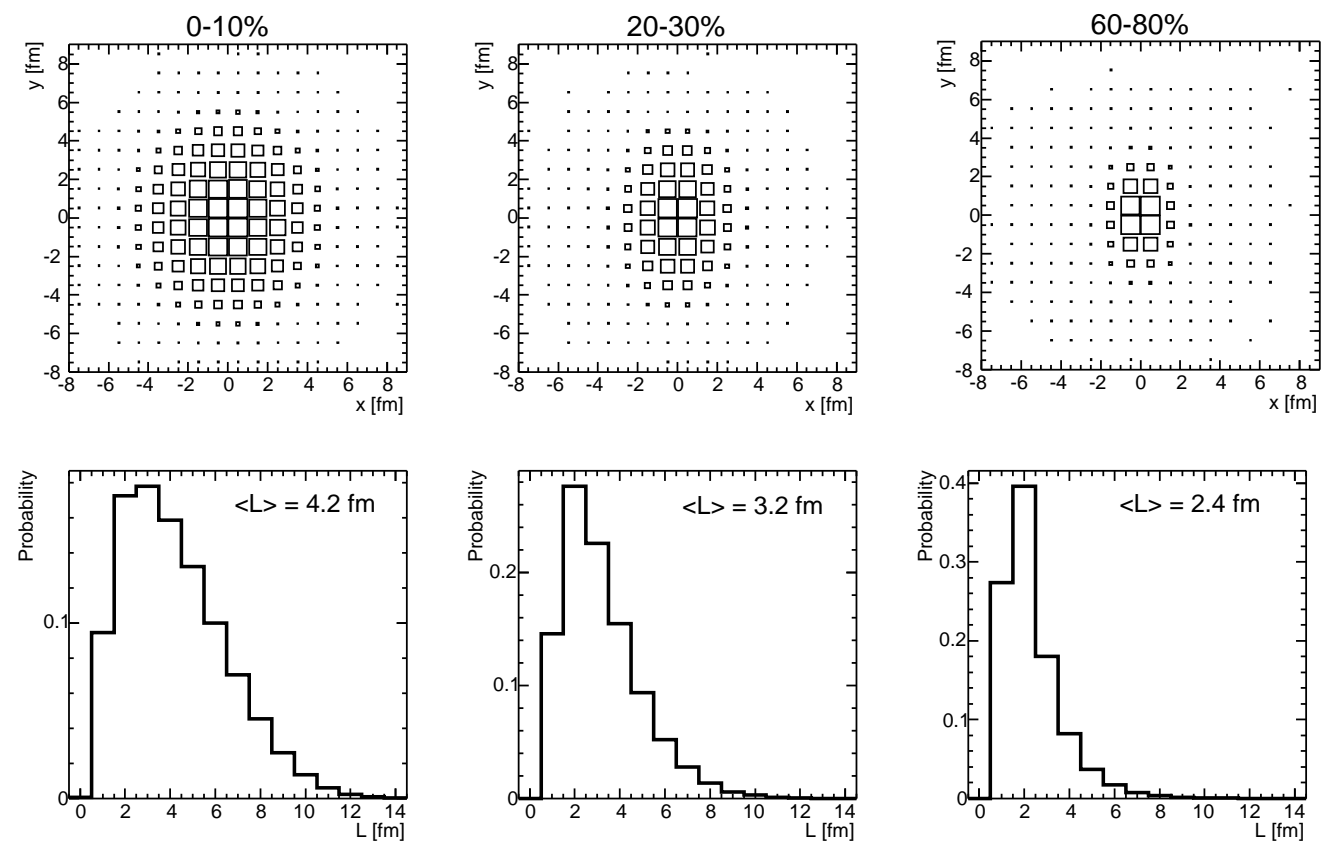

Figure 1: Distributions of parton production points in the transverse plane (upper row) and in-medium path length (lower row) in central, semi-central and peripheral $\mathrm{Au}-\mathrm{Au}$ collisions.

Our new approach allows a natural extension from central to peripheral nucleus-nucleus collisions: the idea is to fix the only free parameter, $k$, in order to describe the measured nuclear modification factor in central collisions and then use the impact parameter $(b)$ dependence of the product $T_{\mathrm{A}} T_{\mathrm{B}}(x, y ; b)$. We translate, by means of the Glauber model, the experimental definition of the centrality classes in terms of fractions of the geometrical cross section to a range in $b$ and, within such range, we sample, for every loop of the chain reported at the beginning of this section, a value of $b$ according to the $b$-dependence of the average number of binary collisions, $\mathrm{d}\left\langle N_{\text {coll }}^{\mathrm{AB}}\right\rangle / \mathrm{d} b$.

For a given centrality class one can compute the distributions of the two more customary and intuitive variables $L$ and $\hat{q}$, derived for every parton from $\omega_{c}$ and $R$ using Eqs. (21) and (3) as:

$$
L=R / \omega_{c}=2 I_{1} / I_{0} \quad \text { and } \quad \hat{\mathrm{q}}=2 \omega_{\mathrm{c}}^{2} /(\mathrm{LR})=\mathrm{I}_{0}^{2} /\left(2 \mathrm{I}_{1}\right) \text {. }
$$

We point out that the resulting definition of $L$ is, as necessary, independent of $k$. Furthermore, it is the same we used in Ref. [19. Note that $\hat{q}$ is proportional to $k$. In Fig. 1 we report for illustration the distributions of 
parton production points in the transverse plane and of in-medium path lengths, in central (0-10\%), semi-central (20-30\%) and peripheral (60-80\%) $\mathrm{Au}-\mathrm{Au}$ collisions. We will show the $\hat{q}$ distributions for different centralities in the next section (in Fig. (4), after we have fixed the scale $k$.

\section{Energy-loss sampling}

In the third step of the chain, we use the numerical routine provided in Ref. [1] to obtain the energy-loss probability distribution for given $\omega_{c}, R$ and parton species (quark or gluon). According to this distribution, we sample an energy loss $\Delta E$ to be subtracted from the parton transverse momentum, $p_{\mathrm{t}}^{\prime}=$ $p_{\mathrm{t}}-\Delta E$. The quenching weights are calculated in the eikonal approximation, where the energy of the parton is infinite $\left(E=p_{\mathrm{t}}=\infty\right)$. Therefore, when the realistic case of finite-energy partons is considered, a significant part of the energy-loss probability distribution $P(\Delta E)$ lies above the parton energy $E$, in particular for large values of $\omega_{c}$ and $R$, or equivalently, of $\hat{q}$ and $L$. The energy loss, under the constraints introduced by the finite parton energies, is sampled following two approaches:

- Reweighted: truncate $P(\Delta E)$ at $\Delta E=E$ and renormalize it to 1 by dividing out the factor $\int_{0}^{E} \mathrm{~d} \epsilon P(\epsilon)$. The Monte Carlo implementation of this approach is: sample $\Delta E$ from the original $P(\Delta E)$; if $\Delta E>E$, sample another $\Delta E$; iterate until a $\Delta E \leq E$ is sampled.

- Non-reweighted: truncate $P(\Delta E)$ at $\Delta E=E$ and add the $\delta$-function $\delta(\Delta E-E) \int_{E}^{\infty} \mathrm{d} \epsilon P(\epsilon)$ to it. The total integral of $P$ is, in this way, maintained equal to 1 . The corresponding Monte Carlo implementation reads: sample an energy loss $\Delta E$ and set the new parton energy to 0 if $\Delta E \geq E$.

The effective energy loss is larger in the non-reweighted case, where partons are 'absorbed' by the medium with a probability $\int_{E}^{\infty} \mathrm{d} \epsilon P(\epsilon)$. As we will see in the next section, the difference can be quite large for low $p_{\mathrm{t}}$ and sufficiently-large transport coefficients. It is argued [11, 24] that the difference in the values of the observables for the two approaches illustrates the theoretical uncertainties. Along the lines of what is done in a recent work [13] developed in parallel to the present study, we display our model results as a band delimited by a solid line representing the non-reweighted case (larger quenching) and a dashed line representing the reweighted case (smaller quenching). While a more theory-oriented discussion on this uncertainty can be found in Ref. [13], we shall come back to this point in our conclusions with a few simple considerations. 


\section{Results}

Nuclear modification factor in $\mathbf{A u}-\mathbf{A u}$ at $\sqrt{s_{\mathrm{NN}}}=200 \mathrm{GeV}$

We start by presenting the results on $R_{\mathrm{AA}}\left(p_{\mathrm{t}}\right)$ in central $\mathrm{Au}-\mathrm{Au}$ collisions at $\sqrt{s_{\mathrm{NN}}}=200 \mathrm{GeV}$ obtained using constant in-medium path length and transport coefficient (left-hand panel of Fig. 2). The data on charged hadrons and neutral pions from STAR [1] and PHENIX [2] are reported with statistical and $p_{\mathrm{t}}$-dependent systematic errors shown by the bars on the data points and $p_{\mathrm{t}}$-independent normalization errors shown by the bars centred at $R_{\mathrm{AA}}=1$. The model results are shown by the lines: for all hadrons, with $\hat{q}=1 \mathrm{GeV}^{2} / \mathrm{fm}$ and $L=6 \mathrm{fm}$, (solid line) and for hadrons coming from quarks and from gluons, separately, with $\hat{q}=0.75 \mathrm{GeV}^{2} / \mathrm{fm}$ and $L=6 \mathrm{fm}$, (dashed and dot-dashed lines). In order to compare our results to those in Ref. [1], we use the same parameters and treat the finite-energy constraint in the non-reweighted case. The two lines obtained with $\hat{q}=0.75 \mathrm{GeV}^{2} / \mathrm{fm}$ and $L=6 \mathrm{fm}$ agree with those reported in Fig. 20 of Ref. [11]. Since the high- $p_{\mathrm{t}}$ hadron spectrum at RHIC energies is mainly coming from quarks ${ }^{1}$, which lose less energy than gluons, a larger $\hat{q}$ of $\simeq 1 \mathrm{GeV}^{2} / \mathrm{fm}$ is necessary to match the measured $R_{\mathrm{AA}}$, when a realistic quarks-to-gluons ratio is used.

Before moving to the parton-by-parton approach of PQM outlined in the previous sections, it is very instructive to show the model results obtained using a constant transport coefficient and the Glauber-based path-length distribution for $0-10 \%$ central collisions (displayed in the bottom-left panel of Fig. (1). We need $\hat{q} \simeq 15 \mathrm{GeV}^{2} /$ fm to describe the data with the model band delimited by the reweighted and non-reweighted cases (right-hand panel of Fig. (2). When going from a constant $L=6 \mathrm{fm}$ to a realistic distribution, the transport coefficient has to be increased by more than one order of magnitude, because there are many partons with small path lengths of $2-3 \mathrm{fm}$ that can be quenched only if the medium is very dense. It is interesting to note that $R_{\mathrm{AA}}$ is clearly increasing with $p_{\mathrm{t}}$ when a constant length is used, while it is flatter with the full distribution. This is due to the presence of a long tail in the $L$ distribution, up to $12 \mathrm{fm}$ : only high-energy partons can fully 'exploit' this tail, while low-energy ones are just completely stopped by the medium after a few fm, so that the 'effective' average length increases with the parton energy. We note that between the non-reweighted and reweighted approach

\footnotetext{
${ }^{1} \mathrm{At} \sqrt{s}=200 \mathrm{GeV}$, with CTEQ $4 \mathrm{~L}$ parton distribution functions 21], gluons dominate the parton $p_{\mathrm{t}}$ distribution up to about $20 \mathrm{GeV}$. However, since quarks fragment harder than gluons, high- $p_{\mathrm{t}}$ hadrons are mostly produced from quark fragmentation. Using KKP fragmentation functions [22], we find that $75 \%$ of the hadrons with $p_{\mathrm{t}}>5 \mathrm{GeV}$ come from quark fragmentation and $25 \%$ from gluon fragmentation.
} 

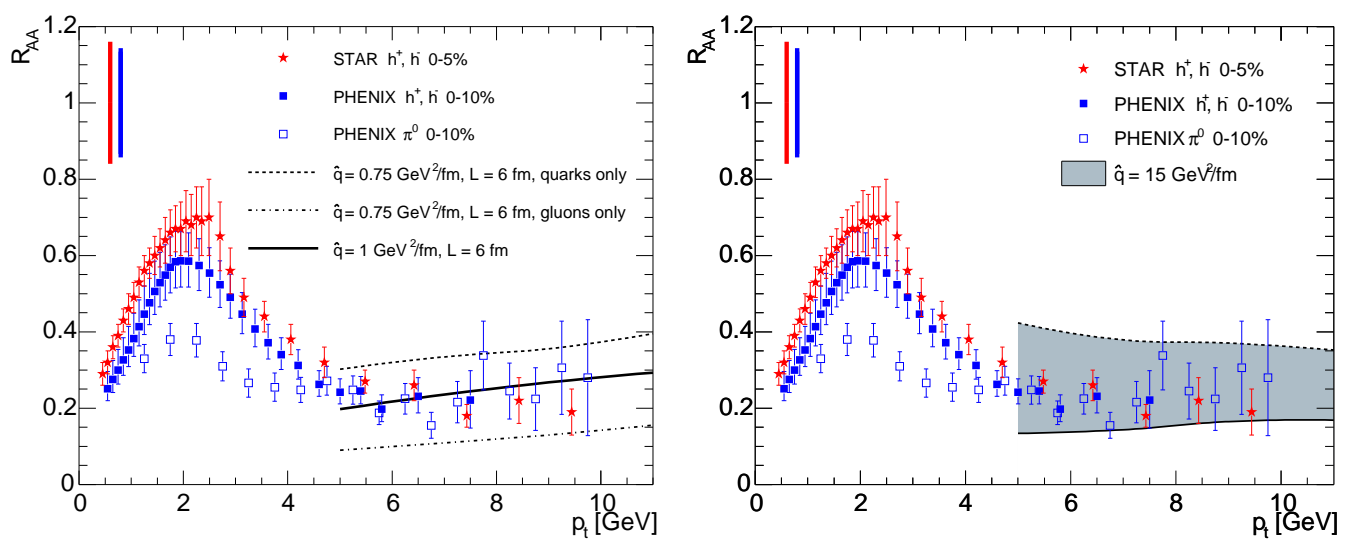

Figure 2: $R_{\mathrm{AA}}\left(p_{\mathrm{t}}\right)$ for central $\mathrm{Au}-\mathrm{Au}$ collisions at $\sqrt{s_{\mathrm{NN}}}=200 \mathrm{GeV}$. STAR and PHENIX data are reported with statistical and $p_{\mathrm{t}}$-dependent systematic errors (bars on the data points) and $p_{\mathrm{t}}$-independent systematic errors (bars at $R_{\mathrm{AA}}=1$ ). Model results for constant $\hat{q}$ and $L$ (left-hand panel) and for constant $\hat{q}$ and Glauber-based $L$ distribution (right-hand panel) are reported. In the right-hand plot and in all the following figures the shaded band is delimited by non-reweighted case (solid) and reweighted case (dashed).

to the parton finite-energy constraint there is a difference of about a factor 2 in the magnitude of $R_{\mathrm{AA}}$, but also a difference in the slope versus $p_{\mathrm{t}}$, which is slightly positive for non-reweighted and slightly negative for reweighted.

Using a constant transport coefficient of $15 \mathrm{GeV}^{2} / \mathrm{fm}$ and a realistic $L$ distribution, the measured hadron suppression can be fairly well described for $p_{\mathrm{t}} \gtrsim 5 \mathrm{GeV}$ (at lower $p_{\mathrm{t}}$ we do not apply the model, as initial-state effects and in-medium hadronization, that we do not include, might play an important role). Remarkably, our result agrees with that obtained in Ref. 13], where the same quenching weights and a simplified collision geometry with effective nuclei (cylindrical density profile instead of the Wood-Saxon we use) are coupled to a leading-order perturbative QCD calculation. The $R_{\mathrm{AA}}$ band is found to have similar $p_{\mathrm{t}}$-dependence (rather flat) and width. Numerically, the extracted value of $\hat{q}$ is $\simeq 7 \mathrm{GeV}^{2} / \mathrm{fm}$ in Ref. [13], much smaller than our $15 \mathrm{GeV}^{2} / \mathrm{fm}$. However, this is not an inconsistency, since the value of $\alpha_{s}$ used in the calculation of the quenching weights is 0.5 in Ref. [13] and 0.3 here, and the scale of the energy loss is set by the product $\alpha_{s} \hat{q}$ (see Eq. (5)).

In order to address the centrality dependence of the high- $p_{\mathrm{t}}$ suppression, we shift to the parton-by-parton approach. The result for central collisions is shown in Fig. 3. with the scale parameter $k=5 \times 10^{6} \mathrm{fm}$, corresponding to $\langle\hat{q}\rangle \simeq 14 \mathrm{GeV}^{2} / \mathrm{fm}$, the model band is very similar to that reported in 


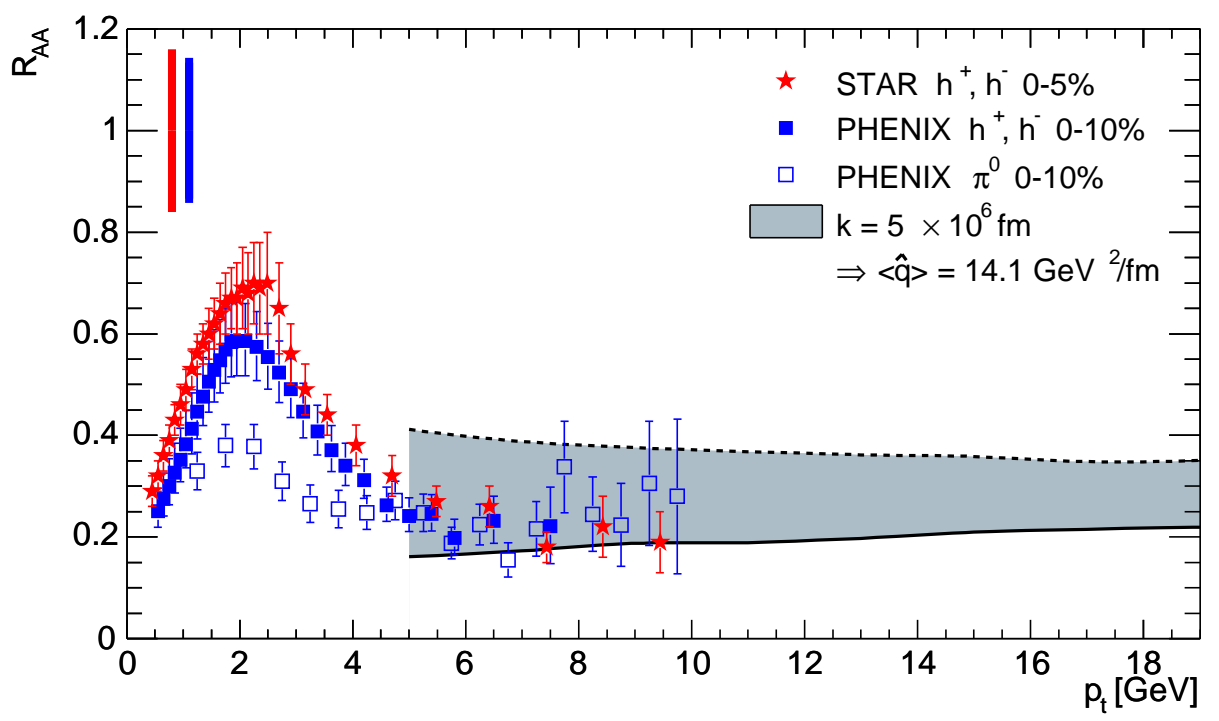

Figure 3: $R_{\mathrm{AA}}\left(p_{\mathrm{t}}\right)$ for central $\mathrm{Au}-\mathrm{Au}$ collisions at $\sqrt{s_{\mathrm{NN}}}=200 \mathrm{GeV}$. The model band is obtained with a parton-by-parton calculation of $\omega_{c}$ and $R$. The average transport coefficient is $14 \mathrm{GeV}^{2} / \mathrm{fm}$.

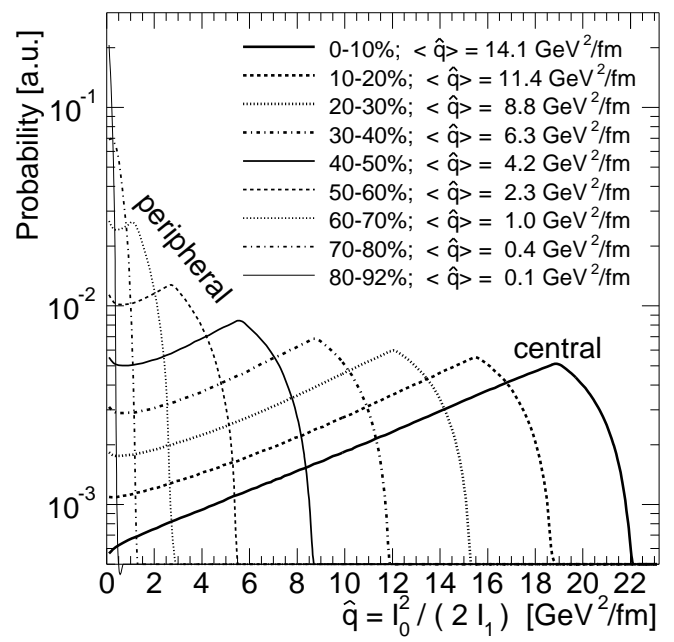

Figure 4: Distributions of $\hat{q}$, calculated from Eq. (14), for different centralities; the $k$ parameter is fixed to the value that allows to describe $R_{\mathrm{AA}}$ for the most central collisions.

the right-hand panel of Fig. 2 for $\hat{q}=15 \mathrm{GeV}^{2} / \mathrm{fm}$ and the $L$ distribution. Now we vary the centrality, without changing the scale $k$. Figure 4 shows the 


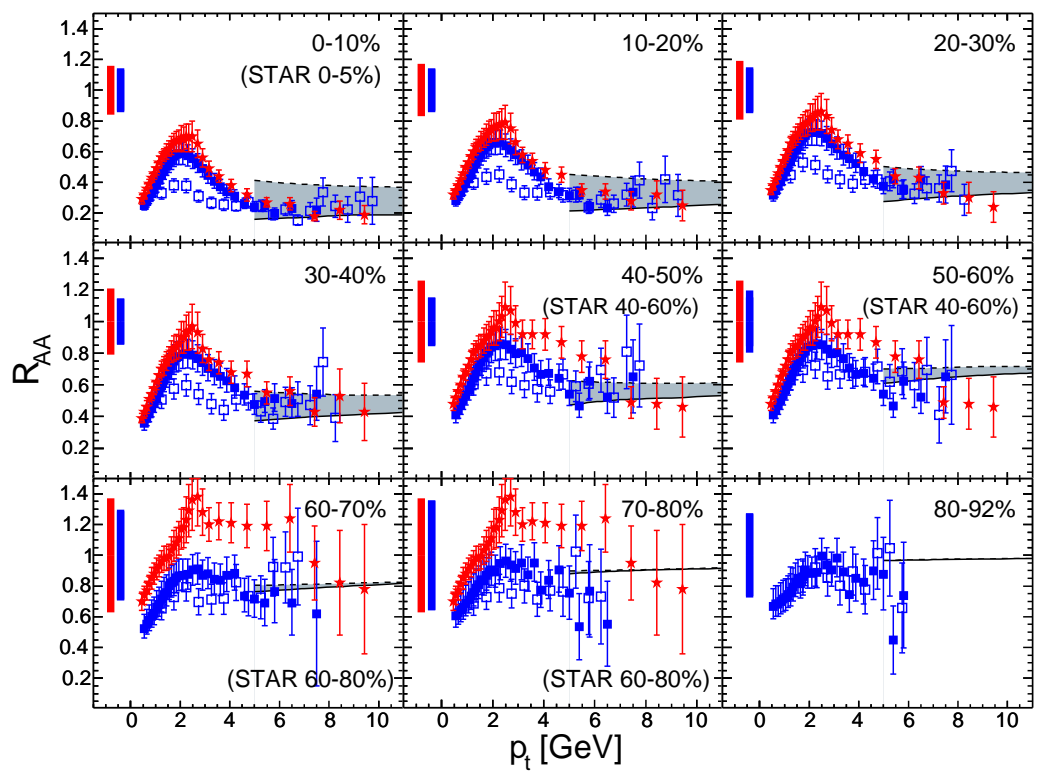

Figure 5: $R_{\mathrm{AA}}\left(p_{\mathrm{t}}\right)$ for different centralities. Data are PHENIX charged hadrons (closed squares) and $\pi^{0}$ (open squares) and STAR charged hadrons (stars).

distributions of $\hat{q}$, calculated from Eq. (14), for different centrality bins. The $\hat{q}$ variation within a given bin reflects the different parton production points, hence different medium densities encountered. The rightmost value refers to partons originating in the centre of the collision system. The model nuclear modification factors compared to STAR [1] and PHENIX 2] data ranging from $0-5 \%$ to $80-92 \%$, are reported in Fig. 5. We note that the theoretical uncertainty band is narrower for semi-central and peripheral collisions, where, due to the smaller size and density of the medium, the probability to have $\Delta E>E$ in the quenching weights becomes marginal.

Our results follow the centrality increase of the measured $R_{\mathrm{AA}}$. This is more conveniently visualized in the left-hand panel of Fig. 6] where we show the average $R_{\mathrm{AA}}$ in the range $4.5<p_{\mathrm{t}}<10 \mathrm{GeV}$ plotted as a function of the number of participant nucleons, $N_{\text {part }}$, obtained from the Glauber model. Data are taken from Refs. [1, 2]. 

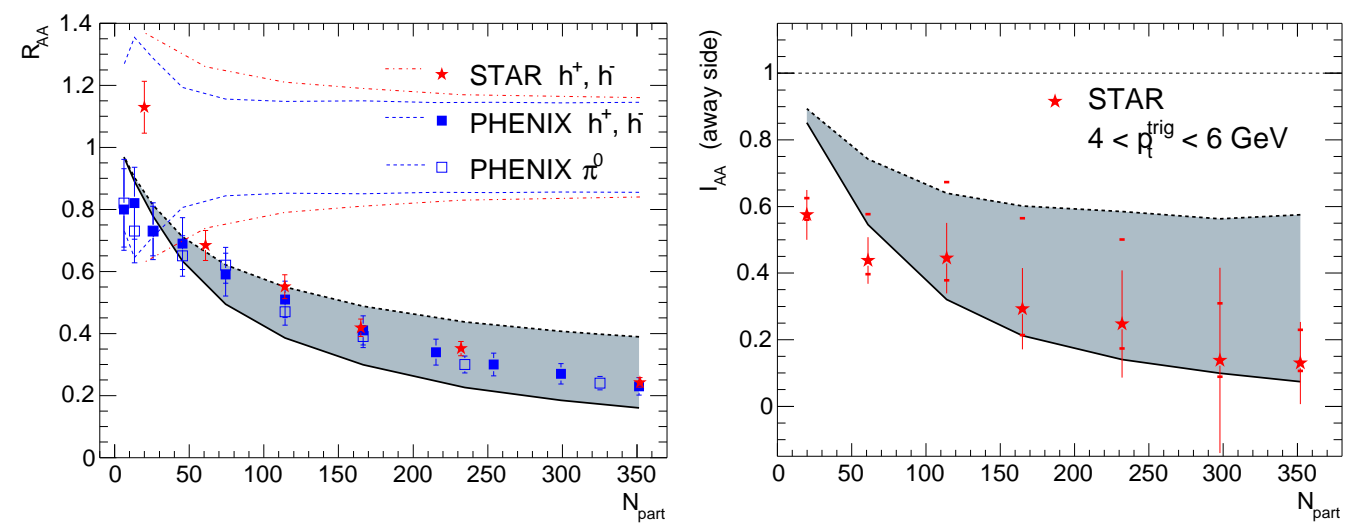

Figure 6: Average $R_{\mathrm{AA}}$ in the range $4.5<p_{\mathrm{t}}<10 \mathrm{GeV}$ (left-hand panel) and $I_{\mathrm{AA}}$, defined in the text, for the away-side jet (right-hand panel) as a function of collision centrality, expressed by the number of participants, $N_{\text {part }}$. For $R_{\mathrm{AA}}$, the error bars are the sum of statistical and $p_{\mathrm{t}}$-dependent systematic errors and the bands centred at $R_{\mathrm{AA}}=1$ are the $p_{\mathrm{t}}$-independent normalization errors, in percents, for STAR (dot-dashed) and PHENIX (dashed). For $I_{\mathrm{AA}}$, the statistical (bars) and systematic (ticks) errors are shown.

\section{Back-to-back correlations}

By generating pairs of back-to-back partons, we can study the centrality dependence of the disappearance of the away-side jet. This effect is usually quantified using the correlation strength [25]

$$
D_{\mathrm{AA}}=\int_{p_{\mathrm{t}}^{\min }}^{p_{\mathrm{t}, 1}} \mathrm{~d} p_{\mathrm{t}, 2} \int_{\Delta \phi>\Delta \phi^{\min }} \mathrm{d} \Delta \phi \frac{\mathrm{d}^{3} \sigma_{\mathrm{AA}}^{h_{1} h_{2}} / \mathrm{d} p_{\mathrm{t}, 1} \mathrm{~d} p_{\mathrm{t}, 2} \mathrm{~d} \Delta \phi}{\mathrm{d} \sigma_{\mathrm{AA}}^{h_{1}} / \mathrm{d} p_{\mathrm{t}, 1}}
$$

for an associated hadron $h_{2}$ with $p_{\mathrm{t}, 2}$ in the opposite azimuthal direction of a trigger hadron $h_{1}$ with $p_{\mathrm{t}, 1}$. The STAR data [3] are for trigger particles with $4<p_{\mathrm{t}, 1}<6 \mathrm{GeV}$ and associated particles with $p_{\mathrm{t}, 2}>p_{\mathrm{t}}^{\min }=2 \mathrm{GeV}$ and $p_{\mathrm{t}, 2}<p_{\mathrm{t}, 1}$ with $\Delta \phi \equiv\left|\phi_{1}-\phi_{2}\right|>\Delta \phi^{\mathrm{min}}=130^{\circ}$. The correlation strength is then corrected for combinatorial background and azimuthal anisotropy of particle production in non-central collisions 3]. The correlation strength in nucleus-nucleus relative to pp collisions defines the suppression factor:

$$
I_{\mathrm{AA}}=\frac{D_{\mathrm{AA}}}{D_{\mathrm{pp}}}
$$

We generate pairs of partons with the same initial $p_{\mathrm{t}}$ and separated in azimuth by $\Delta \phi=180^{\circ}$. Then, we calculate $\omega_{c}$ and $R$ for each parton and apply energy 
loss and fragmentation. We count as trigger particle every hadron $h_{1}$ with $4<p_{\mathrm{t}, 1}<6 \mathrm{GeV}$ and as associated away-side particle the other hadron $h_{2}$ of the pair, if it is in the range $2 \mathrm{GeV}<p_{\mathrm{t}, 2}<p_{\mathrm{t}, 1}$. We define:

$$
I_{\mathrm{AA}}=\left(\frac{N^{\text {associated }}}{N^{\text {trigger }}}\right)_{\mathrm{w} / \text { energy loss }} /\left(\frac{N^{\text {associated }}}{N^{\text {trigger }}}\right)_{\mathrm{w} / \mathrm{o} \text { energy loss }} .
$$

The right-hand panel of Fig. 6] shows our results for $I_{\mathrm{AA}}$ versus $N_{\text {part }}$, compared to STAR measurements in $\mathrm{Au}-\mathrm{Au}$ collisions at $\sqrt{s_{\mathrm{NN}}}=200 \mathrm{GeV}$, with statistical (bars) and systematic (ticks) errors, from Ref. 3. The magnitude and centrality dependence of the suppression are described without changing the scale parameter value we extracted from $R_{\mathrm{AA}}$ in central collisions.

\section{Azimuthally-differential observables}

For non-central collisions, the nucleus-nucleus overlap profile is asymmetric with respect to the event-plane direction, defined by the line that contains the centres of the two colliding nuclei and the impact parameter vector $\vec{b}$, in the transverse plane. This can be seen in the upper row of Fig. 1] where the event-plane direction is parallel to the $x$ axis. Consequently, the in-medium path length is, on average, larger for partons propagating in the out-of-plane direction (perpendicular to the event plane) than for partons propagating in the in-plane direction (parallel to the event plane).

Due to parton energy loss, the asymmetry in the medium geometry should be reflected in the azimuthal distribution $\mathrm{d} N / \mathrm{d} \phi$ of high $-p_{\mathrm{t}}$ hadrons with respect to the event plane, $\phi=0^{\circ}$. We quantify this effect by calculating:

- the azimuthal anisotropy, as given by the second Fourier coefficient of the $\mathrm{d} N / \mathrm{d} \phi$ distribution, $v_{2}$ [26]; we obtain the value of $v_{2}$ for hadrons in a given $p_{\mathrm{t}}$ range by fitting their azimuthal distribution to the form $a \cdot\left(1+2 v_{2} \cos 2 \phi\right)$;

- $R_{\mathrm{AA}}^{\phi_{0}}\left(p_{\mathrm{t}}\right)$, the nuclear modification factor for hadrons in an azimuthal cone of $45^{\circ}$ centred at the angle $\phi_{0}$ with respect to the event plane; we use $\phi_{0}=0^{\circ}$ (in-plane), $\phi_{0}=90^{\circ}$ (out-of-plane) and $\phi_{0}=45^{\circ}$ (intermediate);

- $I_{\mathrm{AA}}^{\phi_{0}}$ (away side), the nucleus-nucleus away-side correlation strength relative to $\mathrm{pp}$, in the three azimuthal regions defined for $R_{\mathrm{AA}}^{\phi_{0}}$.

The scale parameter $k$ is again kept to the value that allows to match $R_{\mathrm{AA}}$ in central collisions at $\sqrt{s_{\mathrm{NN}}}=200 \mathrm{GeV}$.

Figure 7 (left-hand panel) shows the model results for $v_{2}$ as a function of the transverse momentum, for central $\left(0-10 \%, N_{\text {part }} \approx 320\right)$ and noncentral $\left(20-60 \%, N_{\text {part }} \approx 100\right) \mathrm{Au}-\mathrm{Au}$ collisions, compared to non-central 

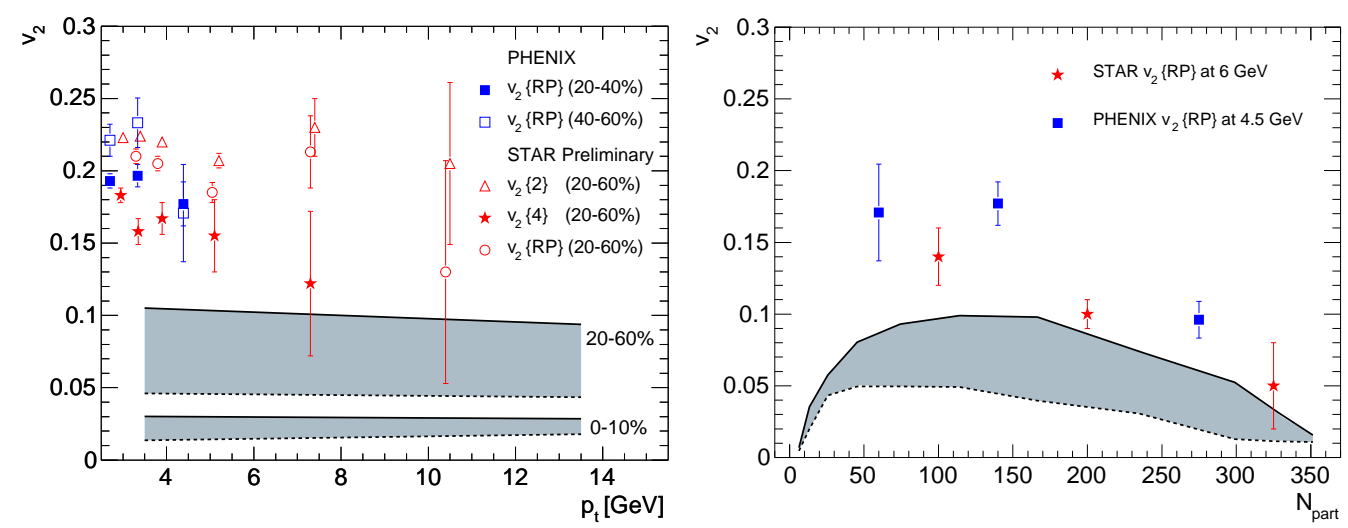

Figure 7: Transverse momentum and centrality dependence of the azimuthal anisotropy $v_{2}$, compared to $v_{2}$ results for charged hadrons from STAR 27, 30] and PHENIX [28] (see text). Only the statistical errors are plotted. Note that, opposed to all other figures, here the model result for the non-reweighted case (solid) is the upper limit of the band and that for the reweighted case (dashed) is the lower one.

experimental measurements on charged hadrons obtained by STAR (preliminary) [27] and PHENIX [28] using three different methods: 2-particle correlations $\left(v_{2}\{2\}\right)$ [29], 4-particle correlations $\left(v_{2}\{4\}\right)$ [29] and reaction plane reconstruction $\left(v_{2}\{\mathrm{RP}\}\right)$. In the right-hand panel of the same figure, the $v_{2}$ centrality dependence from the model is compared to charged hadron data from PHENIX [28, at $p_{\mathrm{t}} \approx 4.5 \mathrm{GeV}$, and from STAR (preliminary) [30, at $p_{\mathrm{t}} \approx 6 \mathrm{GeV}$.

The measured azimuthal anisotropy at intermediate transverse momenta of $4-6 \mathrm{GeV}$ is systematically larger than that generated by parton energy loss in our model, indicating the presence of non-negligible collective flow effects in this momentum range. However, the preliminary STAR measurements at higher $p_{\mathrm{t}}$, shown in the left-hand panel of Fig. [7] suggest that $v_{2}$ might go down to values compatible with those expected from parton energy loss in an azimuthally-asymmetric medium alone. High- $p_{\mathrm{t}}$ data with larger statistics from the recent RHIC Run- 4 will allow to clarify this point. We note that our maximum $v_{2}$ of $0.05-0.10$, for $N_{\text {part }} \approx 100$, is similar to that obtained in other parton energy loss 25 or absorption 12 calculations.

The azimuthal variation of the nuclear modification factor and of the away-side correlations is illustrated in Fig. 8. For $R_{\mathrm{AA}}$ (left-hand panel), we show the two ratios $R_{\mathrm{AA}}^{90} / R_{\mathrm{AA}}^{0}$ (out-of-plane/in-plane) and $R_{\mathrm{AA}}^{45} / R_{\mathrm{AA}}^{0}$ (intermediate/in-plane), averaged over the range $4.5<p_{\mathrm{t}}<10 \mathrm{GeV}$, as 

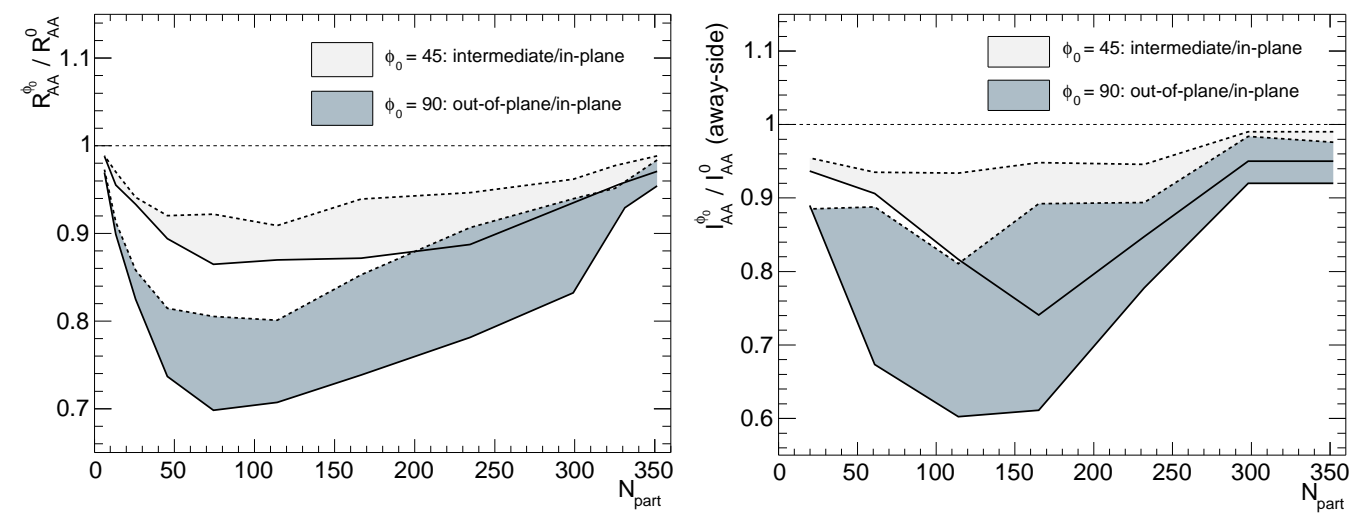

Figure 8: Azimuthal variation of the nuclear modification factor of the awayside correlations. In the left-hand panel, ratios $R_{\mathrm{AA}}^{90} / R_{\mathrm{AA}}^{0}$ (out-of-plane/inplane) and $R_{\mathrm{AA}}^{45} / R_{\mathrm{AA}}^{0}$ (intermediate/in-plane), averaged over the range $4.5<$ $p_{\mathrm{t}}<10 \mathrm{GeV}$. In the right-hand panel, the same ratios for $I_{\mathrm{AA}}$ with trigger conditions as in Fig. 6. Both observables are plotted as a function of $N_{\text {part }}$.

a function of collision centrality $\left(N_{\text {part }}\right)$. As for $v_{2}$, the asymmetry is maximum at $N_{\text {part }} \approx 100$, where the model gives for $R_{\mathrm{AA}}$ a ratio (out-of-plane/inplane) of $\approx 0.75$. Similarly, for the away-side correlation $I_{\mathrm{AA}}$ (right-hand panel), we show the two ratios $I_{\mathrm{AA}}^{90} / I_{\mathrm{AA}}^{0}$ (out-of-plane/in-plane) and $I_{\mathrm{AA}}^{45} / I_{\mathrm{AA}}^{0}$ (intermediate/in-plane). The conditions on the near-side trigger and the associated away-side particles are the same as for Fig. 6. At $N_{\text {part }} \approx 100-150$ the model predicts an away-side correlation strength of about $30 \%$ lower for the out-of-plane relative to the in-plane direction. Both effects are rather strong and their measurement at RHIC would be of great interest.

Nuclear modification factor at $\sqrt{s_{\mathrm{NN}}}=62.4 \mathrm{GeV}$

The recent $\mathrm{RHIC}$ run with $\mathrm{Au}-\mathrm{Au}$ collisions at $\sqrt{s_{\mathrm{NN}}}=62.4 \mathrm{GeV}$ is expected to allow the measurement of the nuclear modification factor for charged hadrons and neutral pions up to transverse momenta of 7-8 GeV. We estimate the leading-particle suppression due to parton energy loss at this lower centre-of-mass energy by using the ansatz done in Ref. [13]: the transport coefficient of the medium is proportional to the initial volume-density of gluons, $n^{\mathrm{g}}$. In the saturation model [31, for collisions of two nuclei with mass number $\mathrm{A}$ at energy $\sqrt{s_{\mathrm{NN}}}$, such density is estimated to scale as

$$
n^{\mathrm{g}} \propto \mathrm{A}^{0.383}\left(\sqrt{s_{\mathrm{NN}}}\right)^{0.574} .
$$




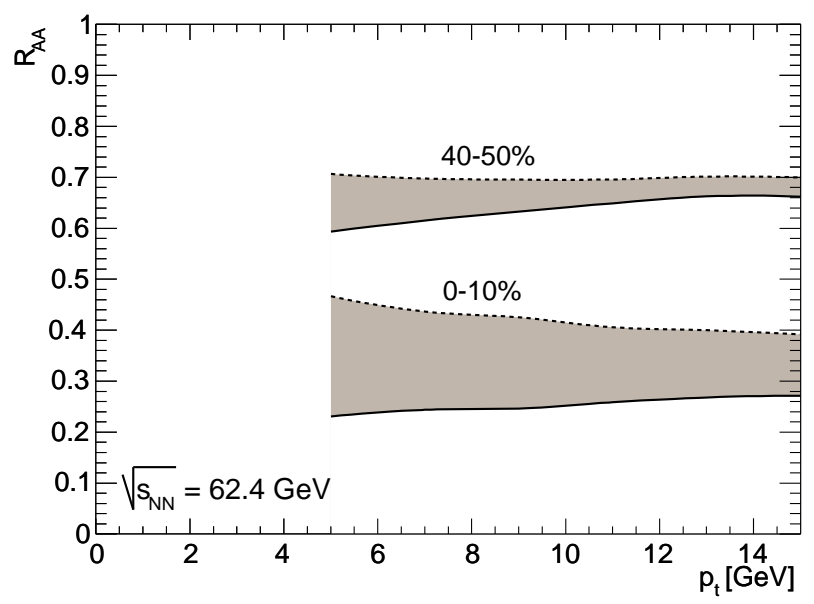

Figure 9: $R_{\mathrm{AA}}\left(p_{\mathrm{t}}\right)$ for central and semi-peripheral $\mathrm{Au}-\mathrm{Au}$ collisions at $\sqrt{s_{\mathrm{NN}}}=62.4 \mathrm{GeV}$.

This gives $n_{\mathrm{Au}-\mathrm{Au}, 62.4 \mathrm{GeV}}^{\mathrm{g}} \simeq 0.5 \times n_{\mathrm{Au}-\mathrm{Au}, 200 \mathrm{GeV}}^{\mathrm{g}}$. Applying this scaling to the $k$ parameter extracted in our model from central collisions at $200 \mathrm{GeV}$, we obtain a transport coefficient distribution with mean value $\langle\hat{q}\rangle \simeq 7 \mathrm{GeV}^{2} / \mathrm{fm}$ in central collisions at $62.4 \mathrm{GeV}$.

We generate hard partons using PYTHIA at $\sqrt{s}=62.4 \mathrm{GeV}$ and use the procedure described in Section [3. The results are shown in Fig. 9] for $p_{\mathrm{t}}>5 \mathrm{GeV}$, we find $R_{\mathrm{AA}} \simeq 0.3$ and $\simeq 0.7$ in central $(0-5 \%)$ and semiperipheral (40-50\%) collisions, respectively. These values are not much larger than those at $\sqrt{s_{\mathrm{NN}}}=200 \mathrm{GeV}$. At smaller $\sqrt{s_{\mathrm{NN}}}$, although the transport coefficient is reduced by a factor of 2 , the increased steepness of the parton transverse momentum distribution determines a stronger effect of energy loss on the nuclear modification factor.

Our results are in agreement with preliminary data from PHENIX [32] for neutral pions up to $p_{\mathrm{t}} \approx 7 \mathrm{GeV}$ in $0-10 \%$ central collisions.

\section{Extrapolation to the LHC}

To compute the expected nuclear modification factor in $\mathrm{Pb}-\mathrm{Pb}$ collisions at the LHC we use PQM with the parton $p_{\mathrm{t}}$ distribution extracted from PYTHIA at $\sqrt{s_{\mathrm{NN}}}=5.5 \mathrm{TeV}$. Scaling the $k$ parameter according to Eq. (18), we have $n_{\mathrm{Pb}-\mathrm{Pb}, 5.5 \mathrm{TeV}}^{\mathrm{g}} \simeq 7 \times n_{\mathrm{Au}-\mathrm{Au}, 200 \mathrm{GeV}}^{\mathrm{g}}$, i.e. $\langle\hat{q}\rangle \simeq 100 \mathrm{GeV}^{2} / \mathrm{fm}$.

We report in Fig. 10 the expected transverse-momentum dependence of $R_{\mathrm{AA}}$ in the range $10<p_{\mathrm{t}}<90 \mathrm{GeV}$ for different centralities. For comparison, the results at $\sqrt{s_{\mathrm{NN}}}=200 \mathrm{GeV}$ are shown as well. In the most central 


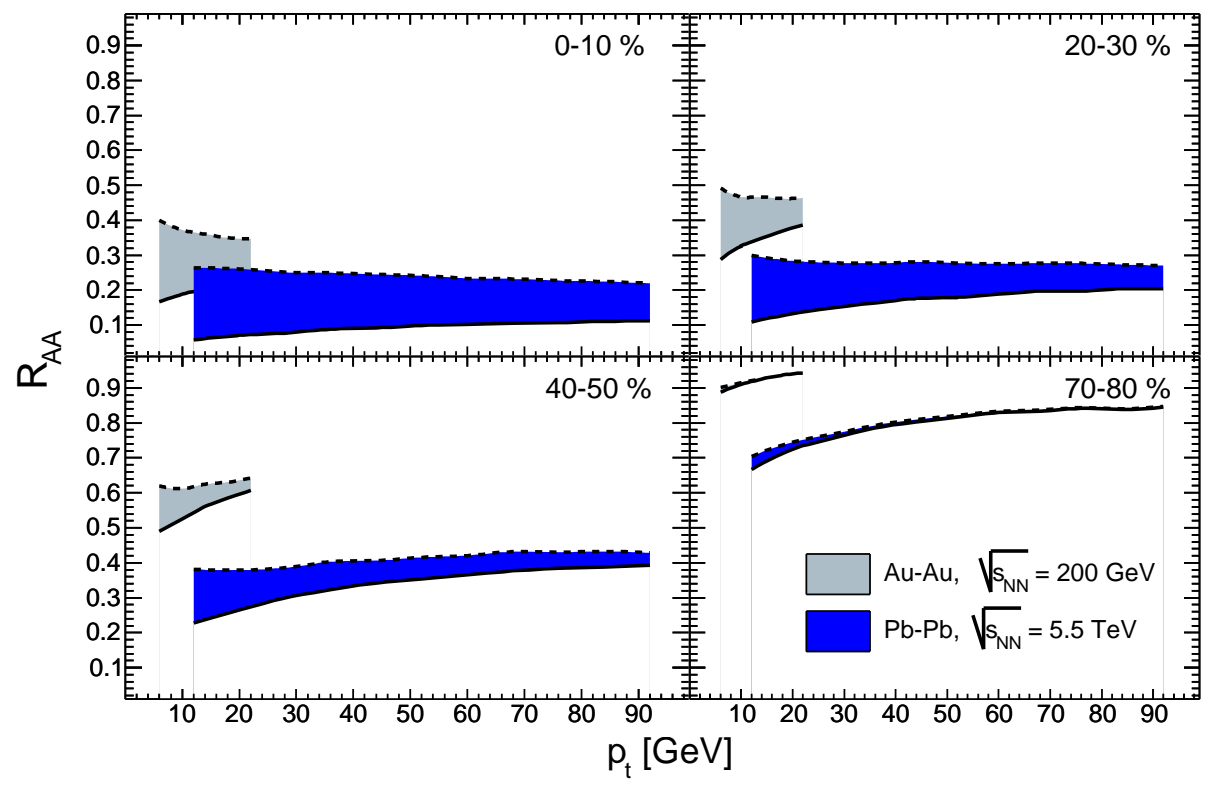

Figure 10: $R_{\mathrm{AA}}\left(p_{\mathrm{t}}\right)$ for different centrality classes in $\mathrm{Pb}-\mathrm{Pb}$ collisions at $\sqrt{s_{\mathrm{NN}}}=5.5 \mathrm{TeV}$ and in $\mathrm{Au}-\mathrm{Au}$ collisions at $\sqrt{s_{\mathrm{NN}}}=200 \mathrm{GeV}$.

collisions $R_{\mathrm{AA}}$ is of $\approx 0.15$, independent of $p_{\mathrm{t}}$. This value is about a factor of 2 smaller than that measured at RHIC for $\sqrt{s_{\mathrm{NN}}}=200 \mathrm{GeV}$. Our result is in agreement, both in the numerical value and in the $p_{\mathrm{t}}$-dependence, with that obtained in Ref. [13] using the same quenching weights and the same $\alpha_{s}\langle\hat{q}\rangle$, while it is quite different from that calculated in Ref. [15], where $R_{\mathrm{AA}}$ is predicted to rise significantly at large transverse momenta: from $0.1-0.2$ at $20 \mathrm{GeV}$ to $0.4-0.6$ at $90 \mathrm{GeV}$. We note that such difference is not likely to be due the fact that we do not include nuclear shadowing effects, since these are expected to determine a rather $p_{\mathrm{t}}$-independent increase of $R_{\mathrm{AA}}$ of about $10 \%$ in the range $25<p_{\mathrm{t}}<100 \mathrm{GeV}$ [14, 15].

\section{Discussion}

\section{High-energy partons from the surface}

The centrality dependence of leading-hadron suppression and back-to-back di-hadron correlations is well described by our model, in which the centrality evolution is purely given by collision geometry. This suggests that the 

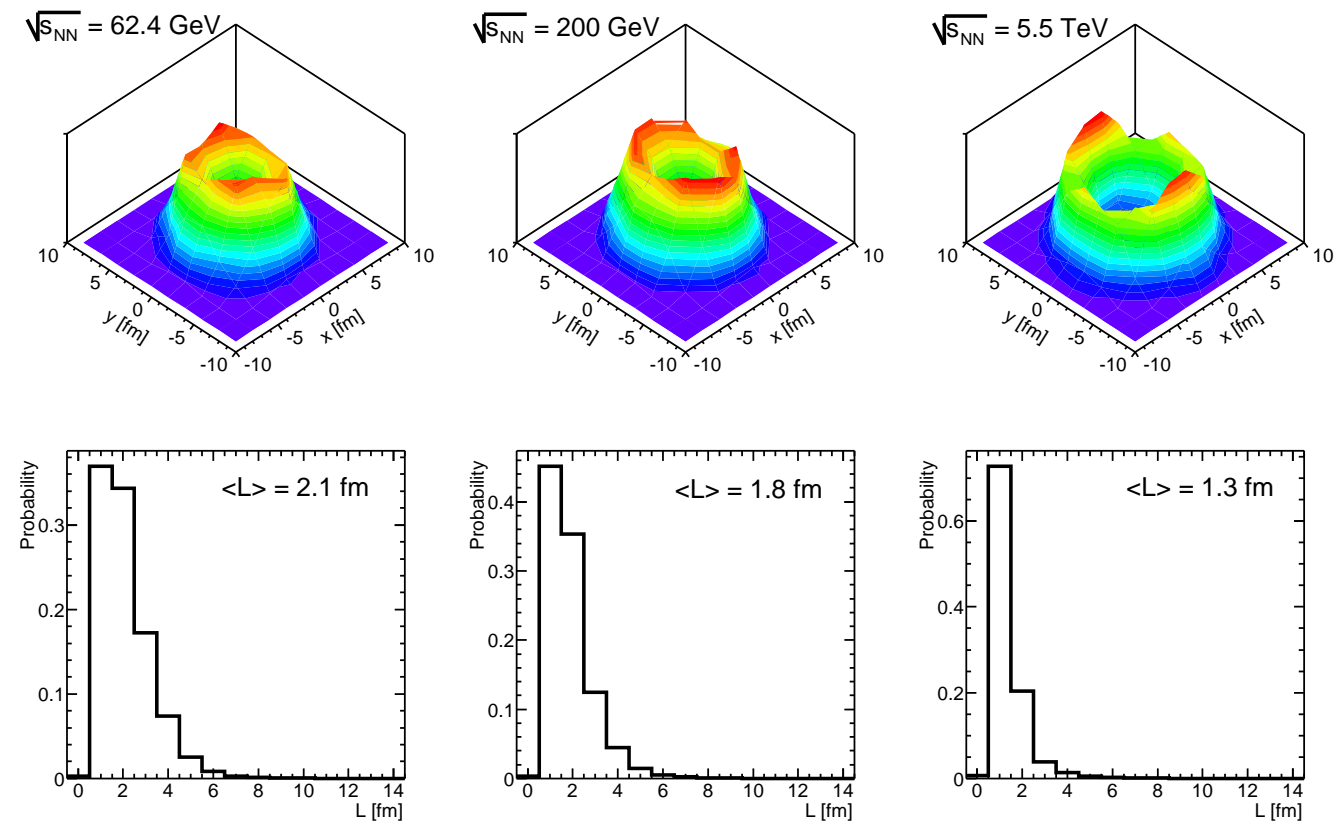

Figure 11: Distributions of parton production points in the transverse plane (upper row) and in-medium path length (lower row) for partons that give hadrons with $p_{\mathrm{t}}>5 \mathrm{GeV}$ in central $\mathrm{Au}-\mathrm{Au}$ collisions at 62.4 and $200 \mathrm{GeV}$ and in central $\mathrm{Pb}-\mathrm{Pb}$ collisions at $5.5 \mathrm{TeV}$. These plots were obtained in the non-reweighted approach.

high-opacity medium formed in $\mathrm{Au}-\mathrm{Au}$ collisions at $\sqrt{s_{\mathrm{NN}}}=200 \mathrm{GeV}$ has initial size and density that decrease from central to peripheral events according to the overlap profile of the colliding nuclei, $T_{\mathrm{A}}(x, y) \times T_{\mathrm{B}}(x, y)$. At the centre of the medium the density is maximum and partons crossing this region are likely to be completely absorbed. Only partons produced close to the surface and propagating outward can escape from the medium with sufficiently high energy to give hadrons above few $\mathrm{GeV}$ in $p_{\mathrm{t}}$. Such an 'emission from the surface' scenario was pictured also in a recent work [12, where the centrality dependence of $R_{\mathrm{AA}}$ and $I_{\mathrm{AA}}$ could be reproduced by a simple model of jet absorption whose only physical ingredient was a Glauber-based nucleus-nucleus overlap profile.

The region from which partons escape from the medium is visualized by plotting the distribution of production points for partons that give a highenergy hadron $\left(p_{\mathrm{t}}>5 \mathrm{GeV}\right)$. This distribution for central $\mathrm{Au}-\mathrm{Au}$ collisions at 62.4 and $200 \mathrm{GeV}$ and $\mathrm{Pb}-\mathrm{Pb}$ collisions at $5.5 \mathrm{TeV}$ is shown in Fig. 11. 


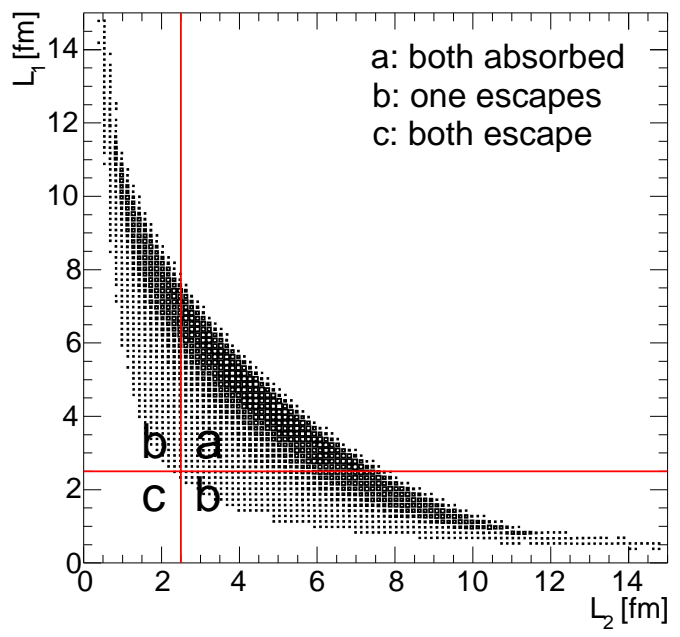

Figure 12: Correlation between path lengths for pairs of partons produced back-to-back in 0-10\% central $\mathrm{Au}-\mathrm{Au}$ collisions.

along with the corresponding path length distribution. The thickness of the escape region is of order $2-3 \mathrm{fm}$ and it decreases as $\sqrt{s_{\mathrm{NN}}}$ increases from intermediate RHIC energy to LHC energy.

It is interesting to try to apply a simple toy model: all partons with a path length $L$ smaller than a maximum length $L_{\text {escape }}^{\max }$ escape from the medium, the others are absorbed. Using the length probability distribution $\mathcal{P}(L)$ and the measured (or expected) $R_{\mathrm{AA}}$ for given collision energy and centrality, $L_{\text {escape }}^{\max }$ can be estimated as $\int_{0}^{L_{\text {escape }}^{\max }} \mathrm{d} \ell \mathcal{P}(\ell)=R_{\mathrm{AA}}$. At $\sqrt{s_{\mathrm{NN}}}=200 \mathrm{GeV}$, we find $L_{\text {escape }}^{\max } \approx 2.5$ fm from central $(0-5 \%)$ to semi-peripheral collisions (40-60\%): in this wide centrality range the simultaneous decrease of system density and volume results in energetic partons being emitted from a shell of constant thickness. For more peripheral collisions the system becomes very diluted and partons can escape from the whole volume $\left(L_{\text {escape }}^{\max } \approx 3.5-4 \mathrm{fm} \approx\right.$ system size). In central collisions at different energies, we find $L_{\text {escape }}^{\max } \approx 3 \mathrm{fm}$ at $\sqrt{s_{\mathrm{NN}}}=62.4 \mathrm{GeV}$ and $L_{\text {escape }}^{\max } \approx 1.5 \mathrm{fm}$ at LHC $\sqrt{s_{\mathrm{NN}}}=5.5 \mathrm{TeV}$.

Remarkably, this absorption toy model allows to reconcile the magnitude of single-particle and away-side correlation suppressions measured in central $\mathrm{Au}-\mathrm{Au}$ at $200 \mathrm{GeV}$, as illustrated in Fig. 12. The figure shows the distribution $L_{1}$ versus $L_{2}$ for pairs of partons (1 and 2) generated back-to-back. Parton pairs produced in the middle of the overlap profile populate the central part of the distribution $\left(L_{1} \sim L_{2}\right)$, while pairs produced closer to the surface are in the two tails $\left(L_{1} \gg L_{2}\right.$ or $\left.L_{1} \ll L_{2}\right)$. We report the two lines $L_{1}=L_{\text {escape }}^{\max }$ and $L_{2}=L_{\text {escape }}^{\max }$, which divide the distribution in three 

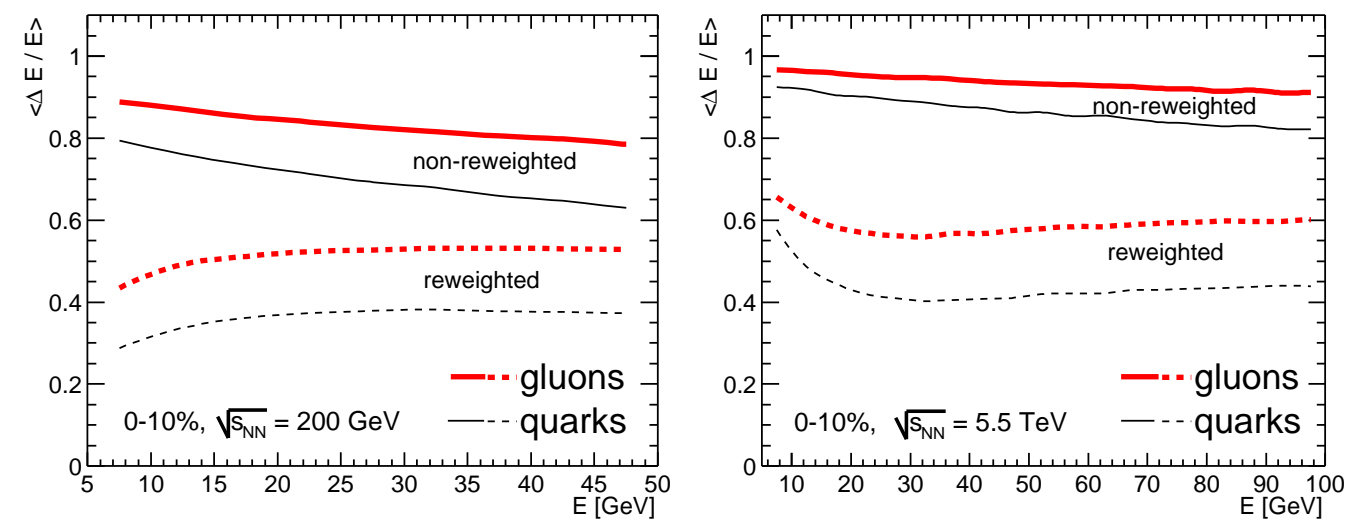

Figure 13: Average relative energy loss versus parton energy for quarks and gluons in central collisions at RHIC and LHC energies for the non-reweighted and reweighted case.

parts: (a) for $L_{1,2}>L_{\text {escape }}^{\max }$ both partons are absorbed, (b) for $L_{1(2)}<L_{\text {escape }}^{\max }$ and $L_{2(1)}>L_{\text {escape }}^{\max }$ only one of the two partons escape the medium, and (c) for $L_{1,2}<L_{\text {escape }}^{\max }$ both partons escape. With the value $L_{\text {escape }}^{\max }=2.5 \mathrm{fm}$, extracted from the measured $R_{\mathrm{AA}}$, the third part of the distribution (c) is empty: it never happens that both partons can escape, in agreement with the value compatible with zero measured by STAR for $I_{\mathrm{AA}}$.

\section{Energy-loss saturation}

The strong parton absorption suggests that we are in a saturation regime of the energy loss, $\Delta E / E \rightarrow 1$, as almost all hard partons produced the inner core are thermalized $(\Delta E / E=1)$ before escaping the medium. Indeed, the average relative energy loss, $\langle\Delta E / E\rangle$ (from the Monte Carlo), shown versus parton energy $E$ in Fig. 13 for central collisions at $\sqrt{s_{\mathrm{NN}}}=200$ and $5500 \mathrm{GeV}$, is almost saturating to unity for gluons (70-80\%) and it is very large also for quarks (50-70\%). Due to the fact that gluons are closer to energy-loss saturation than quarks, the ratio of gluon to quark $\langle\Delta E / E\rangle$ is much smaller than the Casimir ratio $C_{R}^{\mathrm{g}} / C_{R}^{\mathrm{q}}=2.25$ expected from Eq. (5). Furthermore, since absorption (and, hence, saturation) is more significant for small- $E$ partons, or, in other words, large- $E$ partons can exploit larger energy losses, the genuine BDMPS $\Delta E / E \propto 1 / E$ is replaced by a rather $E$-independent effective $\Delta E / E$. It is important to point out here that the $p_{\mathrm{t}^{-}}$ independent nuclear modification factor obtained in our model, in agreement with $\mathrm{RHIC}$ data above $\approx 5 \mathrm{GeV}$, is a natural consequence of this saturation 

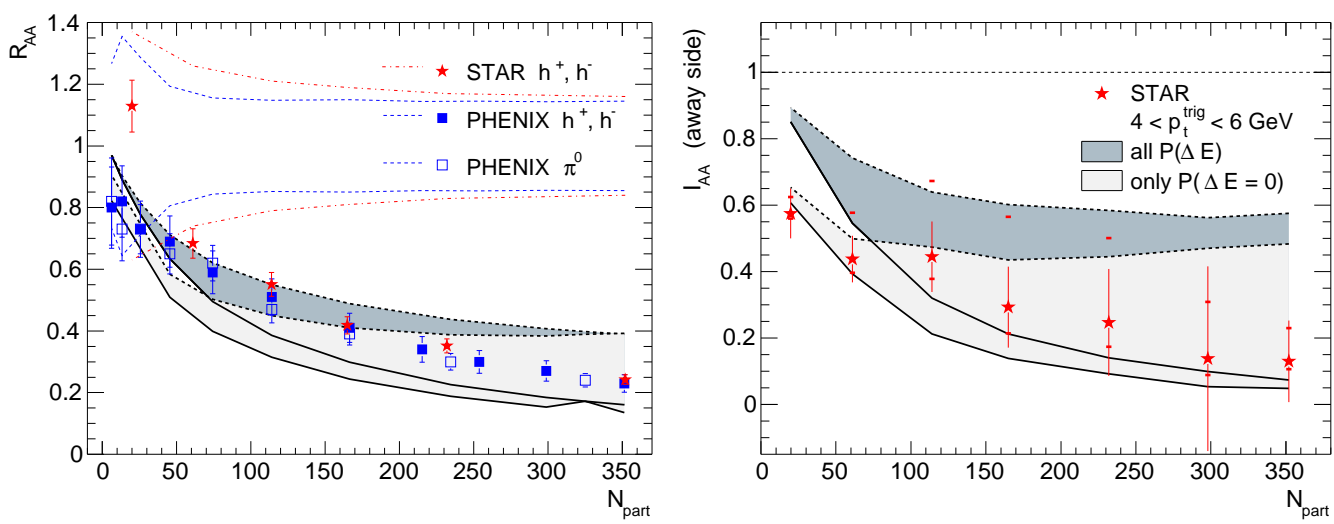

Figure 14: Average $R_{\mathrm{AA}}$ in the range $4.5<p_{\mathrm{t}}<10 \mathrm{GeV}$ (left-hand panel) and $I_{\mathrm{AA}}$ for the away-side jet (right-hand panel) as a function of the number of participants. The data points and errors are the same as in Fig. 6. Here the model results obtained using either zero or maximum energy loss, as explained in the text, (labelled 'only $P(\Delta E=0)$ ', lighter band) are shown together with those obtained with the standard procedure (labelled 'full $P(\Delta E)^{\prime}$, darker band).

scenario.

As the average relative energy loss is close to one, at least for the nonreweighted case, we are not very sensitive to the shape of the continuous part of the quenching weights in Eq. (6) $), p(\Delta E)$. Rather, the energy-loss probability is dominated by the discrete part, the probability to have no medium-induced radiation, $p_{0}$. In order to quantify this statement, we repeat the calculation with a modified PQM version: in the quenching procedure consider the parton as absorbed whenever the sampled energy loss $\Delta E>0$. That is, we have either no energy loss or maximum energy loss. Also in this case, we consider both finite-energy constraint methods, non-reweighted and reweighted. We note that the survival probability is $p_{0}$ for the non-reweighted case and $p_{0} / \int_{0}^{E} \mathrm{~d} \epsilon P(\epsilon)$ for the reweighted case. In Fig. 14 we report $R_{\mathrm{AA}}$ and $I_{\mathrm{AA}}$, calculated with this modified quenching procedure, as a function of the number of participants. For the most central collisions up to 20-30\% the agreement is very good, whereas deviations are clearly visible in $R_{\mathrm{AA}}$ when going to semi-peripheral and peripheral collisions. This confirms that, in central collisions, partons are either completely absorbed or coming from the surface, whereas in non-central collisions the shape of the energy-loss probability distribution plays a role in the description of the data. 


\section{Conclusions}

Most of the present high-momentum observables have been studied using the Parton Quenching Model (PQM) in which hard partons are generated with PYTHIA [20, medium-modified with the quenching weights [11, and hadronized independently via KKP fragmentation functions 22. Using a Glauber approach with Wood-Saxon density profiles of the colliding nuclei, the chain takes into account the realistic spatial distribution of hard parton production points and the amount and density of matter traversed by each parton.

The results show that, if the realistic parton production points and density profiles are taken into account, the ensuing transport coefficient $\hat{q}$ has to acquire very large values, even rather close to the surface. Technically, such values present a yet unsolved theoretical problem since the eikonal approach used in the theory cannot be flawlessly extended to finite (low) parton energies. In the situation, we presented two possibilities:

1. the theoretical treatment is applied regardless of the obvious problem for low-energy partons, which are completely absorbed with a rather large probability (non-reweighted approach);

2. a reweighting procedure is done in order to prevent the possibility to have complete absorption in the medium (reweighted approach).

Our calculations contain only one free parameter that was adjusted to the measured nuclear modification factor in central collisions at $\sqrt{s_{\mathrm{NN}}}=200 \mathrm{GeV}$ at RHIC. The same parameter was then employed to extract the centrality dependence of the nuclear modification factor for hadrons and di-hadrons, and the azimuthal anisotropy parameter $v_{2}$. Some of these observables have

been simulated for $\sqrt{s_{\mathrm{NN}}}=62.4 \mathrm{GeV}$ and $5.5 \mathrm{TeV}$ collisions, scaling only the expected medium densities.

When comparing to experimental results, we observe a tendency for the outright application of the theory, i.e. non-reweighted approach, to fit the $R_{\mathrm{AA}}$ and $I_{\mathrm{AA}}$ centrality dependence reasonably well and, in general, better than the reweighting procedure. The magnitude of $v_{2}$ calculated is about 2 standard deviations below the experimental data taken at $p_{\mathrm{t}} \simeq 4-6 \mathrm{GeV}$; better statistics should be able to give a definitive answer whether the collective elliptic flow subsists at these momenta or not. We have also shown some predictions for the azimuthal variation of leading-particle suppression and jet-like correlations. We note that as a consequence of the azimuthal anisotropy for high- $p_{\mathrm{t}}$ particles simulated in the present approach the lowenergy particles 'radiated' by quenched partons might contribute a $v_{2}$ of the 'opposite sign' in the low- $p_{\mathrm{t}}$ region. Thus, the parton quenching at the LHC 
could produce an apparent decrease of the elliptic flow at low $p_{\mathrm{t}}$ with respect to the predictions of hydrodynamic calculations.

We observe that the reweighting 'simulates' a softer transport coefficient, i.e. it de facto allows for some partons to be emitted from the away side, while a simple geometrical toy model excludes the away-side partons once the model has been tuned on the measured $R_{\mathrm{AA}}$. Furthermore, in the reweighted approach, $R_{\mathrm{AA}}\left(p_{\mathrm{t}}\right)$ is larger towards low transverse momenta, see e.g. Fig. 3 , because the survival probability for a parton with energy $E, p_{0} / \int_{0}^{E} \mathrm{~d} \epsilon P(\epsilon)$, increases when $E$ decreases. This feature appears to be unphysical, or at least non-intuitive. Clearly, the full treatment of the difficulties encountered here should be tackled theoretically in a more complete way.

The inspection of the production-point distribution for energetic partons escaping the medium and of the average relative energy loss suffered by quarks and gluons in central collisions at top RHIC energy depicts the dense medium in the nuclear overlap region as a black disc: either the partons are absorbed or they escape from a thin shell close to the surface.

The present model, applied to the LHC, gives the interesting result that the $R_{\mathrm{AA}}$ value is essentially constant with $p_{\mathrm{t}}$, and very low, up to the highest parton energies. Our prediction differs substantially from others obtained for the LHC [15]. Namely, the black disk effect, which requires a large $\hat{q}$, extends the strong suppression up to very high transverse momenta. This scenario would amount to decrease the number of high-energy jets by almost an order of magnitude and it should be considered in the future planning of experimental studies.

\section{Acknowledgements}

The authors gratefully thank U.A. Wiedemann for help in the formulation of the parton-by-parton calculation of the quenching parameters, $\omega_{c}$ and $R$, A. Morsch for having provided the Glauber model code, D. d'Enterria and C.A. Salgado for useful comments on the manuscript. Fruitful and stimulating discussions with F. Antinori, N. Armesto, K.J. Eskola, H. Honkanen, K. Šafař́k, C.A. Salgado, J. Schukraft and R. Stock are acknowledged.

\section{References}

[1] J. Adams et al., STAR Coll., Phys. Rev. Lett. 91 (2003) 172302 [arXiv:nucl-ex/0305015]. 
[2] S.S. Adler et al., PHENIX Coll., Phys. Rev. Lett. 91 (2003) 072301 [arXiv:nucl-ex/0304022]; Phys. Rev. C69 (2004) 034910 [arXiv:nucl-ex/0308006].

[3] C. Adler et al., STAR Coll., Phys. Rev. Lett. 90 (2003) 082302 [arXiv:nucl-ex/0210033].

[4] J. Adams et al., STAR Coll., Phys. Rev. Lett. 91 (2003) 072304 [arXiv:nucl-ex/0306024].

[5] S.S. Adler et al., PHENIX Coll., Phys. Rev. Lett. 91 (2003) 072303 [arXiv:nucl-ex/0306021].

[6] M. Gyulassy and X.N. Wang, Nucl. Phys. B420 (1994) 583 [arXiv:nucl-th/9306003].

[7] R. Baier, Yu.L. Dokshitzer, A.H. Mueller, S. Peigné and D. Schiff, Nucl. Phys. B483 (1997) 291 [arXiv:hep-ph/9607355]; B484 (1997) 265 [arXiv:hep-ph/9608322]; R. Baier, Yu.L. Dokshitzer, A.H. Mueller and D. Schiff, Phys. Rev. C58 (1998) 1706 [arXiv:hep-ph/9803473]; Nucl. Phys. B531 (1998) 403 [arXiv:hep-ph/9804212].

[8] B.G. Zakharov, JETP Lett. 63 (1996) 952 [arXiv:hep-ph/9607440].

[9] M. Gyulassy, P. Lévai and I. Vitev, Nucl. Phys. B571 (2000) 197 [arXiv:hep-ph/9907461]; $\quad$ Phys. Rev. Lett. 85 (2000) 5535 [arXiv:nucl-th/0005032]; Nucl. Phys. B594 (2001) 371 [arXiv:nucl-th/0006010].

[10] U.A. Wiedemann, Nucl. Phys. B588 (2000) 303 [arXiv:hep-ph/0005129].

[11] C.A. Salgado and U.A. Wiedemann, Phys. Rev. D68 (2003) 014008 [arXiv:hep-ph/0302184].

[12] A. Drees, H. Feng and J. Jia, arXiv:nucl-th/0310044.

[13] K.J. Eskola, H. Honkanen, C.A. Salgado and U.A. Wiedemann, in preparation.

[14] K.J. Eskola and H. Honkanen, Nucl. Phys. A713 (2003) 167 [arXiv:hep-ph/0205048].

[15] I. Vitev and M. Gyulassy, Phys. Rev. Lett. 89 (2002) 252301 [arXiv:hep-ph/0209161]. 
[16] R. Baier, Yu.L. Dokshitzer, A.H. Mueller and D. Schiff, JHEP 0109 (2001) 033 [arXiv:hep-ph/0106347].

[17] R.J. Glauber and G. Matthiae, Nucl. Phys. B21 (1970) 135.

[18] C.W. deJager, H. deVries and C. deVries, Atom. Data Nucl. Data Tabl. 14485.

[19] A. Dainese, Eur. Phys. J. C33 (2004) 495 [arXiv:nucl-ex/0312005].

[20] T. Sjöstrand et al., Computer Phys. Commun. 135 (2001) 238 [arXiv:hep-ph/0010017].

[21] H.L. Lai et al., CTEQ Coll., Phys. Rev. D55 (1997) 1280 [arXiv:hep-ph/9606399].

[22] B.A. Kniehl, G. Kramer and B. Pötter, Nucl. Phys. B582 (2000) 514 [arXiv:hep-ph/0010289].

[23] S.S. Adler et al., PHENIX Coll., Phys. Rev. Lett. 91 (2003) 241803 [arXiv:hep-ex/0304038].

[24] U.A. Wiedemann, Nucl. Phys. A690 (2001) 731 [arXiv:hep-ph/0008241].

[25] X.N. Wang, arXiv:nucl-th/0405017.

[26] A.M. Ponzaker and S.A. Voloshin, Phys. Rev. C58 (1998) 1671.

[27] A. Tang for the STAR Coll., presented at the Quark Matter 2004 Conference, Oakland, January 11-17, 2004.

[28] S.S. Adler et al., PHENIX Coll., Phys. Rev. Lett. 91 (2003) 182301 [arXiv:nucl-ex/0305013].

[29] C. Adler et al., STAR Coll., Phys. Rev. C66 (2002) 034904 [arXiv:nucl-ex/0206001].

[30] K. Filimonov for the STAR Coll., Nucl. Phys. A715 (2003) 737.

[31] K.J. Eskola, K. Kajantie, P.V. Ruuskanen and K. Tuominen, Nucl. Phys. B570 (2000) 379 [arXiv:hep-ph/9909456].

[32] T. Sakaguchi for the PHENIX Coll., presented at the RHIC \& AGS Annual Users' Meeting, Brookhaven, May 10-14, 2004. 